\title{
Strengthening adverse drug reaction reporting in Nepal
}

\author{
Nisha Jha1, Devendra Singh Rathore ${ }^{2}$, Pathiyil Ravi Shankar ${ }^{3}$, Sudesh Gyawali ${ }^{4}$, Mohamed Azmi Hassali ${ }^{5}$, \\ Mohammed Alshakka ${ }^{6}$, Thamir M. Alshammri ${ }^{7}$
}

Lecturer, Department of Clinical Pharmacology and Therapeutics, KIST Medical College, Imadol, Nepal, ${ }^{2}$ Professor and Principal, L.R. Institute of Pharmacy, Himachal Pradesh, ${ }^{3}$ Professor, Pharmacology, Xavier University School of Medicine, Aruba, Kingdom of the Netherlands, ${ }^{4}$ Assistant Professor, Department of Pharmacology, Manipal College of Medical Sciences, Pokhara, Nepal, ${ }^{5}$ Professor, School of Pharmaceutical Sciences, University Sains Malaysia, Malaysia, ${ }^{6}$ Assistant Professor, Department of Pharmaceutics and Clinical Pharmacy, Faculty of Pharmacy, Aden University, Yemen, ${ }^{7}$ Assistant Professor, Department of Clinical Pharmacy, College of Pharmacy, University of Hail, Saudi Arabia

\section{A B S T R A C T}

Pharmacovigilance in Nepal is still in infancy. Till date only healthcare professionals are involved and the problem of underreporting is seen. The national pharmacovigilance centre is located in the national regulatory authority of Nepal, i.e. Department of Drug Administration (DDA). Lack of adequate human resources for managing the pharmacovigilance program in Nepal has been a limitation for the growth of pharmacovigilance activities. Currently, there is neither any involvement of community pharmacists in Adverse Drug Reaction (ADR) reporting process nor any involvement of consumers for the same. This paper reviews the current status of pharmacovigilance and mentions possible benefits of involving consumers or patients in the existing pharmacovigilance program. A systematic review were conducted by searching different databases like PubMed, Google scholar, EMBASE, NepJOL, and Scopus. This study also describes the role of healthcare professionals in ADR reporting, possible reasons for underreporting of ADRs, regulatory perspectives and benefits of involving consumers in pharmacovigilance.
Access this article online Website:

http://nepjol.info/index.php/AJMS DOI: 10.3126/ajms.v6i4.11659

\section{INTRODUCTION}

Medicines are a major component of the modern health care system and can be considered as a 'double edged sword' having both beneficial as well as harmful effects on human beings. Within this context, concern has been raised worldwide about the increasing number of adverse effects caused by drugs. Studies have shown that adverse drug reactions (ADRs) are a major cause of hospitalization and death all over the world. ${ }^{1,2}$ Monitoring ADRs and any other drug related problems is called 'Pharmacovigilance'. The World Health Organization (WHO) defines an ADR as "a response to a drug which is harmful and unintended, and which occurs at doses normally used in man for the prophylaxis, diagnosis, or therapy of disease or for the modification of body functions". ${ }^{3}$

The broader aim of pharmacovigilance is ensuring safe use of medicines and serving as the quality control system for society with regard to use of medicines. Every country should have its own pharmacovigilance program as there are differences in the genetic make-up, diet, use of traditional medicines, cultural differences, methods for manufacturing medicines and many other factors. ${ }^{4}$ To this effect, systematic reviews were conducted by searching different databases like Pubmed, Google scholar, Embase, NepJOL, and Scopus. Different articles were searched in context to Nepal and other developing countries for getting more information on pharmacovigilance and consumer pharmacovigilance. The key words used were adverse drug reactions, Nepal, consumer pharmacovigilance and pharmacovigilance.

Pharmacovigilance in Nepal is still in its infancy. Nepal became a member of the World Health Organization Uppsala Monitoring Centre in 2007. ${ }^{5}$ The national pharmacovigilance centre coordinates with seven centres in Nepal of which five are situated in the 
Kathmandu valley while one each are located in the eastern and western regions of the country. These centres collect ADR reports from health care professionals and forward them to the Department of Drug Administration (DDA) in Kathmandu from where the reports are sent to the Uppsala Monitoring Centre in Sweden which serves as a centre for international service and scientific research towards patient safety using an online program called Vigiflow. ${ }^{6}$ Despite the program having been in operation for over seven years pharmacovigilance (PV) is not mentioned in documents like the policy of hospitals, health institutions, pharmaceutical industries, public health programs and other organizations in the country till date. This highlights the urgent need to include and address pharmacovigilance in Nepal's national policies and regulations. Pharmacovigilance is being addressed in the National Medicine Policy which is under review and is a welcome development. ${ }^{7}$

In Nepal, there has been a sharp increase in the use of both allopathic medicines and also complementary medicines. ${ }^{7}$ This increases the risk of drug-drug interactions between allopathic and complementary medicines and highlights the urgent and increasing need for pharmacovigilance activities. DDA should explore opportunities to collect and collate reports with regard to various health products like medicines, biologics, vaccines and medical devices. The country should adopt international reporting standards for collecting, analysing and understanding possible adverse drug reactions from medicines as well as from medical devices.

The distribution of the pharmacovigilance centres does not cover the entire country and at present there is only one centre each for the eastern and western development region and no centres for the mid-western and far-western regions. ${ }^{6}$ Five centres are located at Kathmandu, which is a part of central region.

The existing system for monitoring ADRs in Nepal depends on voluntary reporting from doctors, pharmacists, nurses and health assistants. Unfortunately, many health care professionals are not aware of the existing system for pharmacovigilance in Nepal. ${ }^{8}$ They lack information about the system for monitoring and reporting ADRs, forms available for ADR reporting and there is no mechanism for periodic update and information sharing about recent ADRs among health care professionals in hospitals, health centres and the community. Underreporting of ADRs is a major limitation as regional centres are functioning with inadequate human and financial resources. ${ }^{9}$ There is a lack/shortage of personnel responsible for conducting pharmacovigilance in peripheral hospitals and health centres.
Role of healthcare professionals in Pharmacovigilance Pharmacists could be in a unique position to report ADRs either on their own or with the help of other health care professionals to reduce the risks of ADRs by detecting, reporting and assessing any suspected ADRs. ${ }^{10}$ They can also educate, suggest and encourage physicians, nurses and other health care professionals for reporting ADRs. ${ }^{11}$ Till date, however, very few hospitals have trained pharmacists to do this job. ${ }^{7}$ Also the pharmacy curriculum followed in schools in Nepal may not adequately prepare pharmacists for this important responsibility. ${ }^{12}$

In regional centres shortage a lack of dedicated personnel for pharmacovigilance. Pharmacists and others working in the centres are also involved in a number of other responsibilities like teaching, running the hospital pharmacy and administrative responsibilities. Lack of motivation and fear among health care professionals especially considering the violent response to patient deaths and injury in many hospitals could be a factor responsible for underreporting. ${ }^{7}$ Another reason for underreporting can be the high patient doctor ratio. The patient: pharmacist ratio is even higher. In 2011 there were 10,197 (3.64/10,000 population) medical and dental doctors, 32,846 (11.71/10,000 population) nursing and midwifery personnel and $731(0.261 / 10,000$ population) licensed pharmacists in Nepal. ${ }^{13}$ Studies have revealed major lack of awareness and perceptions about pharmacovigilance and consumer pharmacovigilance among HCPs. ${ }^{8}, 10,12$ As HCPs could play an important role in educating their patients and are regarded as learned and respected persons in local communities it is important that they have proper knowledge about medicines and provide proper medical care to their patients.

Many healthcare professionalare were unaware of the existence of ADR reporting forms and the reporting process. This could be a contributing factor towards underreporting by HCPs shown by previous studies. ${ }^{8,12}$ Increased patient load can be a factor responsible for health professionals providing less time to patients and not being able to convey all the required information. Additionally, many medicines are available from medicine shops without a doctor's prescription which encourages self-medication and may cause irrational use of medicines, especially antibiotics. ${ }^{14}$ Pharmacists and others working in medicine shops (community pharmacies) are key stakeholders for ensuring safe and rational use of medicines.

\section{Regulatory perspectives}

At present, only one focal pharmacovigilance officer with a post graduate degree in pharmacy is managing the pharmacovigilance program in the national pharmacovigilance centre with the help of a technical 
assistant with a master's degree in pharmacy. This technical assistant maintains the records of ADR reports from all the regional pharmacovigilance centres. The focal person is In-charge of the drug information unit and manages pharmacovigilance as one of the activities. Overall in-charge is the director general of the DDA but since he is the head of the regulatory authority, pharmacovigilance activities are only one of his overall responsibilities. There are no definite guidelines developed till date for establishing, running and assessing the performances of all the seven regional centres in Nepal. There is no particular job responsibility defined for the person responsible for pharmacovigilance in the existing regional pharmacovigilance centres.

Feedback regarding the reported ADRs from the regional centres is provided by the Uppasala Monitoring Centre (UMC) to the national centre and then the feedback is forwarded to all the respective regional pharmacovigilance centres. Feedback is about the reporting processes and issues related to quality of the reports. The drug information unit of DDA publishes the drug bulletin of Nepal quarterly, which contains information on various issues related to medicines. Information about pharmacovigilance till date in these bulletins has been limited to notices about information on the quality of medicines. Drug information unit of DDA has produced some informative posters for consumers regarding information to be known before purchasing any medicines, proper use of metered dose inhalers, allergic reactions caused by use of drugs etc. It also frequently publishes some notices in the local newspapers for creating awareness about the use of medicines. These efforts towards educating consumers regarding using medicines safely are limited only to some hospitals and consumers and have not concentrated on one of the important key stake holders in medicine dispensing and use, the community pharmacist. In Nepal, like in other developing countries not all pharmacies have a trained pharmacist. ${ }^{15}$ There are no guidelines developed for healthcare professionals regarding educating patients about adverse drug reactions.

There is no specific legal provision for pharmacovigilance in the national medicines legislation or any other similar policies. Nepal's Drug Act of 1978 and National Drug Policy (NDP) of 1995 address elements of medicine safety and public health. Pharmacovigilance and consumer pharmacovigilance is not yet addressed in the policy documents of many of Nepal's health institutions, industry, medical professional organisations, or public health programs. Pharmaceutical industries are not yet involved in ADR reporting till date. There are no reports from these industries since the inception of pharmacovigilance activities in Nepal. Community pharmacists are involved in ADR reporting in many countries like Norway and Spain but this is an area to be initiated and explored in Nepal. ${ }^{16,17}$

\section{Consumer pharmacovigilance}

Consumer pharmacovigilance is still a new concept in Nepal. There is no provision for reporting of ADRs by consumers and the lay public in the pharmacovigilance program till date in Nepal. Many developed and a few developing countries currently accept patient reports. Consumers can directly report ADRs to a pharmacovigilance centre either by filling in a paper form, through telephone or online. They can also submit ADRs indirectly through consumer organizations in some developed countries. We can also follow this system for ADR reporting by consumers and a separate form should be designed for them. Involving consumers can help to overcome the problem of underreporting of ADRs and reports can be obtained from all age groups of patients. Involving consumers in the current pharmacovigilance system can reinforce ADR reporting systems and can overcome the problem of underreporting.

Establishing a drug information unit in all the hospitals could enable patients and consumers to obtain unbiased information about medicines.

There should be more persons responsible for managing the pharmacovigilance program at a national level and in the regional pharmacovigilance centres. Till now, there are only about thirty five people working at DDA and among them, about 15 are skilled pharmacy manpower having masters in pharmacy degree, and others look after administrative works. Currently, DDA recruits people for pharmaceutical care. The process for recruitment is via a written exam followed by an interview for the candidates. There should be more number of seats for skilled manpower in organizations like the DDA to monitor and run various programs smoothly. Ministry of health and population should take an initiative for forming an executive functional pharmacovigilance advisory committee to look after all pharmacovigilance related functions. Participation of pharmacists in ADR reporting has shown a positive impact on ADR reporting. This has also resulted in improvement in the number and quality of ADR reports and the drug safety monitoring programme..$^{18,19}$

Government should take initiatives in creating more vacancies for pharmacists and should provide more opportunity for pharmacists to be involved in drug safety and surveillance systems.

Incorporating pharmacovigilance in the syllabi of various health care professional courses like, medicine, nursing, pharmacy and others could be a major step. ${ }^{20}$ 
Pharmacovigilance can be strengthened by having a pharmacovigilance centre, which could be an accreditation requirement for medical schools especially in regions which do not presently have a centre.

Vigorous attempts at promoting awareness should be made by framing proper guidelines for various stakeholders like community pharmacies, health sectors, drug manufacturing industries, academics and most importantly the consumers. Strengthening the pharmacovigilance program in Nepal is a vital and cost-effective investment towards ensuring safer use of medicines among the Nepalese population.

\section{Approaches for strengthening the pharmacovigilance} program in Nepal

Framing a guideline for health care professionals for reporting adverse drug reactions could be a major initiative. Till date, DDA has conducted about ten trainings and seminars related to pharmacovigilance since the establishment of the national pharmacovigilance centre. This number should be increased and can be possible only after recruiting more number of personnel in the pharmacovigilance program.

Involvement of consumers in existing pharmacovigilance program by developing a separate form for them and vigorous awareness should be created by a strong collaboration between National Health Education Information and Communication Centre is required to bring consumer education in action. This centre has an integrated approach for advocacy, community mobilization, communication and health service and program promotion activities. Further expansion of the regional pharmacovigilance centres can help in reporting more ADRs from the peripheral region of the country as well. DDA should be more active in disseminating the information collected and ADR reports by the national pharmacovigilance centre via their publication in drug bulletin of Nepal. There should be strict guidelines for establishing a pharmacovigilance centre in terms of manpower and the job responsibilities to look after regional pharmacovigilance centres. A prospective feedback mechanism should be developed for appropriate dissemination of the reported ADRs back to the reporters like health care professionals. This might motivate people to report more ADRs. Lastly, an award and reward system for reporters may work as a solution for underreporting in developing countries like Nepal and there should be no fear of penalties or adverse consequences among healthcare professionals for reporting ADRs.

\section{CONCLUSION}

Till now, there are no plans for risk alleviation caused due to medicines among consumers in Nepal. Initiating consumer pharmacovigilance can be an important beginning made towards strengthening the existing pharmacovigilance systems and can provide an opportunity for consumers to be involved in pharmacovigilance. This study also highlights the approaches for strengthening pharmacovigilance in Nepal.

\section{REFERENCES}

1. Davies EC, Green CF, Taylor S, Williamson PR, Mottram DR and Pirmohamed M. Adverse drug reactions in hospital in-patients: a prospective analysis of 3695 patient-episodes. PLoS One 2009; 4:e4439.

2. Davies EC, Green CF, Mottram DR and Pirmohamed M. Adverse drug reactions in hospitals: a narrative review. Current Drug Safety $2007 ; 2: 79-87$.

3. World Health Organization. Requirements for adverse reaction reporting. Geneva, Switzerland. World Health Organization; 1975.

4. World Health Organization. The importance of pharmacovigilance: Safety monitoring of medicinal products. Geneva, Switzerland: 2002. Retrieved 12/011/2014 from www.who.int/medicinedocs/ collect/medicinedocs/pdf/s4893e/s4893e.pdf.

5. Nepal joins programme. Uppsala reports 2007;36:5-6. Retrieved 12/012/2014 fromwho-umc.org/graphics/24365.pdf.

6. Jha N, Shankar PR, Bajracharya O, Gurung SB and Singh KK. Adverse drug reaction reporting in a pharmacovigilance centre of Nepal. Australasian Medical Journal 2012;5: 268-271.

7. Jha N, Rathore DS, Shankar PR, Thapa BB, Bhuju G and Alshakka M. Need for involving consumers in Nepal's pharmacovigilance system. Australasian Medical Journal 2014; 7(4):191-195.

8. KC Santosh, Tragulpiankit P, Edwards I. Ralph and Gorsanan S. Knowledge about adverse drug reactions reporting among healthcare professionals in Nepal. International Journal of Risk \& Safety in Medicine 2013; 25:1-16.

9. Lopez-Gonzalez E, Herdeiro MT and Figueiras A. Determinants of underreporting of adverse drug reactions: a systematic review. Drug Safety 2009; 32:19-31.

10. Palaian S, Izham MIM and Mishra P. Evaluation of the knowledge, attitude and practices on adverse drug reactions and pharmacovigilance among healthcare professionals in a Nepalese hospital- a preliminary study. International Journal of Pharmacology 2007;6:1.

11. Herdeiro MT, Figueiras A, Polonia $\mathrm{J}$ and Gestal-Otero JJ. Physicians' attitudes and adverse drug reaction reporting: a case-control study in Portugal. Drug Safety 2005; 28(9):825 833.

12. Palaian S, Ibrahim $\mathrm{MI}$ and Mishra P. Health professionals' knowledge, attitude and practices towards pharmacovigilance in Nepal. Pharmacy Practice 2011; 9(4):228-235.

13. Ministry of Health and Population in collaboration of WHO. September 27, 2011.

14. Shankar PR, Partha P and Shenoy N. Self-medication and nondoctor prescription practices in Pokhara valley, Western Nepal: a questionnaire-based study. BMC Family Practice 2002;3:17.

15. Palaian S, Ibrahim MIM and Mishra P. Pattern of adverse drug reactions reported by the community pharmacists in Nepal. Pharmacy Practice 2010;8:201-207.

16. Gedde-Dahl A, Harg P, Stenberg-Nilsen H, Buajordet M, Granas AG and Horn AM. Characteristics and quality of adverse drug reaction reports by pharmacists in Norway. Pharmacoepidemiology and Drug Safety 2007; 16: 999-1005.

17. Marta I, Guadalupe B, Maira BR, Adolfo F, Sonia HD and 
Berta L. Factors that Influence Under-Reporting of Suspected Adverse Drug Reactions among Community Pharmacists in a Spanish Region. Drug Safety 2007; 30:1073-1082.

18. Van Grootheest AC, van Puijenbroek EP and de Jong-van den Berg LT. Contribution of pharmacists to the reporting of adverse drug reactions. Pharmacoepidemiology and Drug Safety 2002; 11:205-210.
19. Van Grootheest K, Olsson S, Couper M and de Jong-van den Berg L. Pharmacists' role in reporting adverse drug reactions in an international perspective. Pharmacoepidemiology and Drug Safety $2004 ; 13: 457-464$.

20. Santosh KC, Tragulpiankit $P$, Gorsanan $P$, Edwards IR and Alam K. Strengthening pharmacovigilance programme in Nepal. Nepal Journal of Epidemiology 2013;31:230-235.

\footnotetext{
Authors Contribution:

NJ and DSR - Designed the study, collected the data, interpreted the data and drafted the manuscript; PRS, SG, AH, MA and TMA - helped in conceptualizing

the study, helped in interpreting the literature collected and reviewed written drafts of the manuscript. All authors have read and approved the final submitted

version of the manuscript.

Source of Support: Nil, Conflict of Interest: None declared.
} 